\title{
Improving range of SPR tunability and extinction efficiency of spheroidal silver nanostructures in graphene environment
}

\author{
SHIVANI BHARDWAJ*D and R P SHARMA \\ Centre for Energy Studies, IIT Delhi, New Delhi 110016, India \\ *Author for correspondence (sshivanni@gmail.com)
}

MS received 20 March 2018; accepted 28 May 2018; published online 3 September 2018

\begin{abstract}
In photovoltaics, the materials having ability to manipulate the optical fields and coupling of energy flow inside the device play a crucial role. In this article, we report the role of graphene environment on spheroid-shaped Ag nanoparticles (NPs) with various shapes and sizes. This study confirms the tunability of surface plasmon resonances (SPRs) and an enhancement in extinction efficiency, derived numerically using discrete dipole approximation (DDA). We have chosen oblate- and prolate-shaped Ag NPs for the numerical experiment and analysed their optical signatures in terms of extinction efficiency and SPR tunability against the quasi-static approximation. The excitation of longitudinal and transversal resonances was also observed because of the asymmetric shape of Ag NPs. All optical responses have been analysed by varying the effective radii and aspect ratio of $\mathrm{Ag} \mathrm{NPs}$, and the thickness of graphene monolayer (from 0.1 to $0.5 \mathrm{~nm}$ ). Tunability of longitudinal resonances has been observed in the 600-833 nm wavelength region, while for transversal resonances, the tunability is in the 450-505 $\mathrm{nm}$ wavelength range. The results represent the effect of graphene environment on the tunability of SPRs with enhanced extinction efficiency. This study could lead to the development of a photovoltaic device with wide range of tunability and enhanced efficiency.
\end{abstract}

Keywords. Discrete dipole approximation; graphene-plasmon interaction; spheroid-shaped Ag NPs; extinction efficiency; graphene monolayer surrounding environment; localized surface plasmon resonances.

\section{Introduction}

Ultra-thin devices and nanostructures are needed to enhance light-matter interaction, lessen the material usage and also allow more efficient charge collection to deal with a nextgeneration photovoltaic device [1-3]. Due to the reduction in optical thickness of the absorber material in such devices, electromagnetic devices such as nano-optic cavities, localized surface plasmons (LSPs) and photonic crystals have been introduced to enhance the absorption mechanism, which leads to higher photocurrent conversion process [1,4,5]. Metal nano-particles (MNPs) that strongly support LSPs are widely utilized in different fields of applications such as ultrafast optical switching [6], biological sensors [6], optical data storage [7], optical tweezers [8], light-emitting diodes [9], surfaceenhanced Raman scattering [10], solar energy conversion [1], etc. Tunability of the plasmonic modes for MNPs highly depends on their shape, size, physical environment, variation of the dielectric functions and one-to-one interaction of the nanoparticles (NPs) [11-13]. Among them, asymmetricshape MNPs have two or three different orthogonal principal axes that possess multiple plasmonic modes due to their large degree of freedom for the coherent excitation of the conductive electrons oscillating along each axis. The longitudinal (along major axis) and transversal (along minor axis) plasmonic resonances are excited by the charge accumulation of oscillating electrons along the respective axes of NPs $[14,15]$. The coupling between the longitudinal and transversal plasmonic modes of the MNPs plays an important role in enhancing the resonant broadband absorption inside the active layer. In previous studies one can observe that the surface plasmon resonance (SPR) tunability of the MNPs is not appropriate, with a limited wavelength range of tunability $[15,16]$. Hence, there is a strong need of an advance material with its unique optical properties that lead to a wide range of SPR tunability for NPs from visible to IR domain. To increase the efficiency of the photovoltaic devices, many of the researchers have used different surrounding environments of MNPs. Nowadays, graphene monolayer (GML) is widely used as a spacer, substrate, coating material and surrounding environment in the solar cell devices due to its fascinating optical and electrical properties [17-20].

Graphene is a flexible, ultimate-thin 2D material that supports its own plasmonic properties. Graphene plasmonics is a unique and attractive field that gives a new generating platform to promote strong light-matter interaction that enables extreme light confinement. The unusual optical properties of graphene depend on its transparency, conductivity, flexibility and strength, which are extremely high and stable at room temperature [19-22]. Due to such fascinating behaviour, graphene shows outstanding optical features that promote tuning over a wide range of SPR in AM1.5 Spectra. 
The optical and electrical properties of graphene are frequently used in photovoltaics to enhance the photon current density of the solar cells.

In this article, we have studied the interaction between spheroid-shaped silver NPs and GML by the DDA technique. Here we have chosen three different types of spheroidal Ag NPs: sphere, oblate and prolate, to identify the significant enhancement in their extinction efficiencies $\left(Q_{\text {ext }}\right)$ and the SPR tunability. The ASR of the proposed nano-geometries is varied from 0.3 to 0.9 . Thickness of surrounding environment of the MNPs $t_{\mathrm{GML}}=0.1 \mathrm{~nm}$ has been considered for the proposed nano-geometries to achieve the optical broadband SPR tunabilty that covers visible to near-IR domain. Modified optical parameters of the silver NPs have been taken from the literature [23,24].

\section{Theory}

\subsection{Computational method}

Discrete dipole approximation (DDA) is one of the most powerful and flexible electro-dynamics numerical techniques that describes optical signatures such as absorption, scattering and extinction efficiency with excellent accuracy of the entire volume for targets of any arbitrary geometry [25-27].

In DDA, the target geometry is treated as an array of $N$ points of dipoles. The location $\left(\overrightarrow{r_{\mathrm{i}}}\right)$, and polarizability $\left(\vec{\alpha}_{\mathrm{i}}\right)$ of the $N$-point dipoles are the essential parameters for the DDA simulations $[12,25,26]$. The dipole moment of the target geometry is expressed as

$$
\begin{aligned}
\vec{p}_{\mathrm{i}} & =\alpha_{\mathrm{i}} \cdot \vec{E}_{\mathrm{i}, \text { loc }} \\
\vec{E}_{\mathrm{i}, \text { loc }} & =\vec{E}_{\mathrm{i}, \text { app }}+\vec{E}_{\mathrm{i}, \text { ind }} \\
\vec{E}_{\mathrm{i}, \text { app }} & =E_{0} e^{i(\vec{k} \cdot \vec{r}-\omega t)} \\
\vec{E}_{\mathrm{i}, \text { ind }} & =-\sum_{j=1}^{N} \vec{A}_{\mathrm{ij}} \cdot p_{\mathrm{j}}
\end{aligned}
$$

where $\vec{k}$ represents the incident wave vector.

$\vec{E}_{\mathrm{i}, \text { loc }}$ is the local electric field established by the applied electromagnetic field $\vec{E}_{\mathrm{i} \text {,app }}$ on the array of point dipoles. $\vec{E}_{\mathrm{i} \text {,ind }}$ is the induced electric field produced by the radiation of all others $N-1$ dipoles acting on the $i$ th individual point dipole. The $A_{\mathrm{ij}}$ with $i \neq j$ is an interaction matrix with $3 \times 3$ matrices as elements, such that $[25,27,28]$

$$
\begin{aligned}
\vec{A}_{\mathrm{ij}} \cdot \vec{p}_{\mathrm{j}}= & \frac{e^{i k r_{\mathrm{ij}}}}{r_{\mathrm{ij}}^{3}}\left\{k^{2} \vec{r}_{\mathrm{ij}}\left(\vec{r}_{\mathrm{ij}} \times \vec{p}_{\mathrm{j}}\right)+\frac{\left(1-i k r_{\mathrm{ij}}\right)}{r_{\mathrm{ij}}^{2}}\right. \\
& \left.\times\left[r_{\mathrm{ij}}^{2} \vec{p}_{\mathrm{j}}-3 \vec{r}_{\mathrm{ij}}\left(\vec{r}_{\mathrm{ij}} \cdot \vec{p}_{\mathrm{j}}\right)\right]\right\}
\end{aligned}
$$

and

$$
\vec{p}_{\mathrm{i}}=\alpha_{\mathrm{i}} \cdot\left(\vec{E}_{\mathrm{i}, \text { app }}-\sum_{j=1}^{N} \vec{A}_{\mathrm{ij}} \cdot p_{\mathrm{j}}\right)
$$

where $r_{\mathrm{ij}}=\left|r_{\mathrm{j}}-r_{\mathrm{i}}\right|$ and $\vec{r}_{\mathrm{ij}}=\vec{r}_{\mathrm{i}}-\vec{r}_{\mathrm{j}}$. Once we know each $p_{\mathrm{i}}$ of the target geometry, it is possible to calculate the extinction, absorption and scattering cross-sections using the following expressions [25,29]:

$$
\begin{aligned}
C_{\mathrm{ext}} & =\frac{4 \pi k}{\left|\vec{E}_{0}\right|^{2}} \sum_{i=1}^{N} \operatorname{Im}\left\{\vec{E}_{\mathrm{i}, \text { inc }}^{*} \cdot \vec{p}_{\mathrm{i}}\right\} \\
C_{\mathrm{abs}} & =\frac{4 \pi k}{\left|\vec{E}_{0}\right|^{2}} \sum_{i=1}^{N}\left\{\operatorname{Im}\left[\vec{p}_{\mathrm{i}} \cdot\left(\alpha_{\mathrm{i}}^{-1}\right)^{*} \vec{p}_{\mathrm{i}}^{*}\right]-\frac{2}{3} k^{3}\left|\vec{p}_{\mathrm{i}}\right|^{2}\right\} \\
C_{\mathrm{sca}} & =C_{\mathrm{ext}}-C_{\mathrm{abs}} \\
Q_{1} & =\frac{C_{1}}{\pi a_{\mathrm{eff}}^{2}}
\end{aligned}
$$

where $C_{1}$ is the optical cross-sectional area of the target geometry and $l$ is the running index of optical cross-sectional area (scattering, absorption and extinction) of the point dipole. $Q_{1}$ is the normalized optical cross-section and $a_{\text {eff }}$ is the effective radius of target geometry.

\subsection{Optical response of graphene}

The optical responses of the graphene are described using the Kubo formula in terms of the surface conductivity [25,30,31]. The frequency-dependent complex-valued surface conductivity of graphene depends on its intra-conductivity $\sigma_{\text {intra }}$ and inter-conductivity $\sigma_{\text {inter }}$. The dynamic surface conductivity can be quantitatively described as $[17,18,21]$

$$
\begin{aligned}
\sigma_{2 \mathrm{D}}(\omega)=\sigma_{\text {intra }}\left(\omega, \mu_{\mathrm{c}}, \Gamma, T\right)+\sigma_{\text {inter }}\left(\omega, \mu_{\mathrm{c}}, \Gamma, T\right) \\
\begin{aligned}
\sigma_{\text {intra }}\left(\omega, \mu_{\mathrm{c}}, \Gamma, T\right)= & -i \frac{e^{2} \mathrm{k}_{\mathrm{B}} T}{\pi \hbar^{2}(\omega-i 2 \Gamma)} \\
\times & {\left[\frac{\mu_{\mathrm{c}}}{\mathrm{k}_{\mathrm{B}} T}+2 \ln \left(\exp \left(-\frac{\mu_{\mathrm{c}}}{\mathrm{k}_{\mathrm{B}} T}\right)+1\right)\right] } \\
\sigma_{\text {inter }}\left(\omega, \mu_{\mathrm{c}}, \Gamma, T\right)= & -i \frac{e^{2}}{4 \pi \hbar} \\
& \times \ln \left[\frac{2\left|\mu_{\mathrm{c}}\right|-\hbar(\omega-i 2 \Gamma)}{2\left|\mu_{\mathrm{c}}\right|+\hbar(\omega-i 2 \Gamma)}\right] \quad(11 \mathrm{c})
\end{aligned}
\end{aligned}
$$

where $\omega$ is the frequency of the incident light, $\Gamma$ is relaxation process rate having $\Gamma \leq 1 \mathrm{meV}, \mu_{\mathrm{c}}$ is the chemical potential of graphene that lies in approximately 50-1000 meV range and 


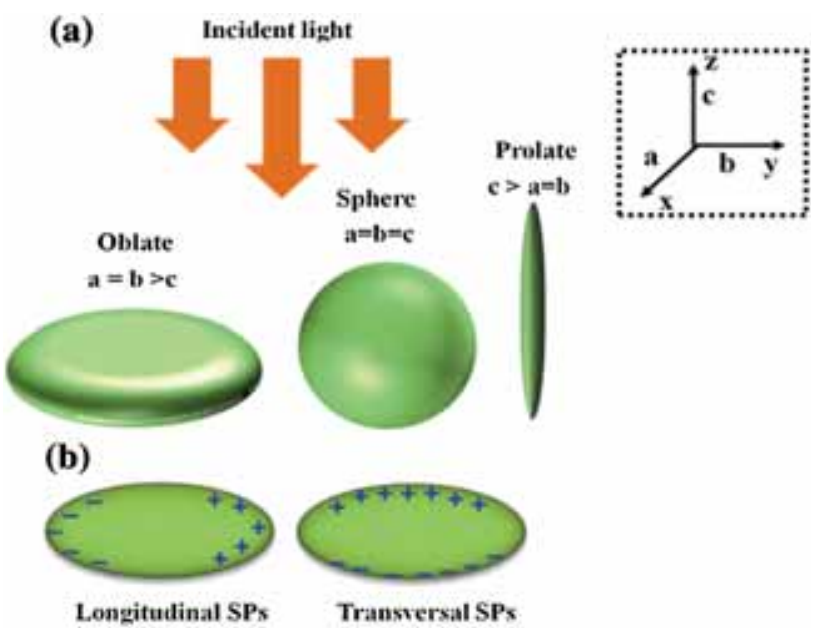

Figure 1. Schematic diagram of (a) 3D spheroid-shaped Ag NPs surrounded by the graphene monolayer (GML) environment, having the dielectric function $\varepsilon(\omega)$. (b) Generalized presentation of charge accumulation for longitudinal and transversal surface plasmons in oblate-shaped Ag NPs.

$\hbar$ is reduced Planck's constant. Further, relative permittivity of graphene $\varepsilon_{\mathrm{g}}(\omega)$ can be obtained by coupling of Ohm's law and Maxwell's equation, which is proportional to its complexvalued surface conductivity as follows $[18,30]$ :

$$
\varepsilon_{\mathrm{g}}(\omega)=\left(\varepsilon_{0}+i \frac{\sigma_{2 \mathrm{D}}(\omega)}{\omega t}\right)
$$

where $\varepsilon_{0}$ is the permittivity of free space and $t$ is the thickness of GML.

A schematic diagram of the spheroid-shaped: oblate $(a=$ $b>c)$, spherical $(a=b=c)$ and probate $(c>a=b)$, $\mathrm{Ag}$ NPs is shown in figure 1a. Figure $1 \mathrm{~b}$ shows the charge accumulation on the surface of the oblate-shaped Ag NP in two different modes of polarization. The charge accumulation highly depends on the shape of the NP. If the charge accumulations are excited along the major axis they are called longitudinal plasmons, while along the minor axis they are known as transversal plasmons. The longitudinal and transversal plasmons lie in the higher and lower wavelength region, respectively, due to the restoring force, which is proportional to the strength of the charge accumulation [32].

\section{Results and discussion}

Plasmon coupling between the Ag NPs and GML surrounding environment provides the guided information to understand basic phenomena of the enhancement in extinction efficiency and the SPR tunabiliy of the Ag NPs embedded in the surrounding medium of graphene. This amplified efficiency plays a significant role in harvesting the absolute light trapping due to the increased effective path length of the incident

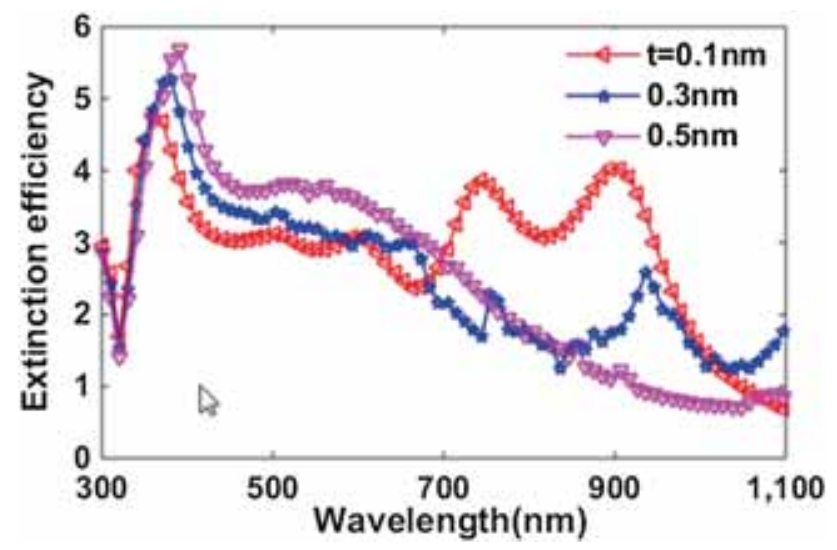

Figure 2. Extinction efficiency $Q_{\text {ext }}$ of spherical Ag NPs at fixed effective radius $a_{\text {eff }}=100 \mathrm{~nm}$ with GML of three different thicknesses $(t=0.1,0.3$ and $0.5 \mathrm{~nm})$ as the surrounding environment.

light, which is responsible for improving the absorption of photons inside ultra-thin silicon substrate.

Figure 2 helps us to understand the modification in the extinction efficiency of sphere-shaped Ag NPs embedded in homogeneous surrounding environments of graphene. Extinction efficiency of Ag NPs has been calculated using equation (10). We have chosen a fixed effective radius $\left(a_{\text {eff }}\right)=$ $100 \mathrm{~nm}$ of the spherical Ag NP to show remarkable resonance effect with different thicknesses of graphene $\left(t_{\mathrm{GML}}\right)$ as the host medium. For $t_{\mathrm{GML}}=0.1 \mathrm{~nm}$, there is the existence of the multiple plasmonic peaks in the lower as well as higher wavelength region simultaneously, which covers the resonant broadband spectrum (extinction efficiency lies in UV-IR domain) other than two thicknesses (0.3 and $0.5 \mathrm{~nm}$ ) of GML as shown in figure 2. Therefore, $t_{\mathrm{GML}}=0.1 \mathrm{~nm}$ of surrounding environment has been considered for further analysis.

In the further discussion, asymmetric-shape NPs (oblate and prolate) have been introduced to analyse the variation in SPR tunability and extinction efficiency compared with symmetrical (spherical-shaped) NPs. The multiple peaks in SPR spectra of asymmetric NPs are observed because of the displacement of the electron cloud, which is non-homogenous about all axes while it is homogeneous for symmetrical NPs.

Figure 3 shows the wavelength-dependent extinction efficiency spectra of oblate $(a=b>c)$ Ag NPs embedded in graphene environment. Figure $3 \mathrm{a}-\mathrm{c}$ presents the resonant broadband of the extinction efficiency spectrum of oblateshaped Ag NPs for different ASR = 0.3, 0.5 and 0.9, respectively, where $a_{\text {eff }}$ of the Ag NPs has been varied from 60 to $100 \mathrm{~nm}$. For oblate-shaped Ag NPs, there is twofold symmetry and hence, two pronounced peaks occur; one lies in higher wavelength regime at $\lambda_{\mathrm{SPR}}=820.8 \mathrm{~nm}$ corresponding to major axis (longitudinal axis) and other lies in the lower wavelength limit at $\lambda_{\mathrm{SPR}}=600 \mathrm{~nm}$ corresponding to minor axis (transverse axis), in the broad 


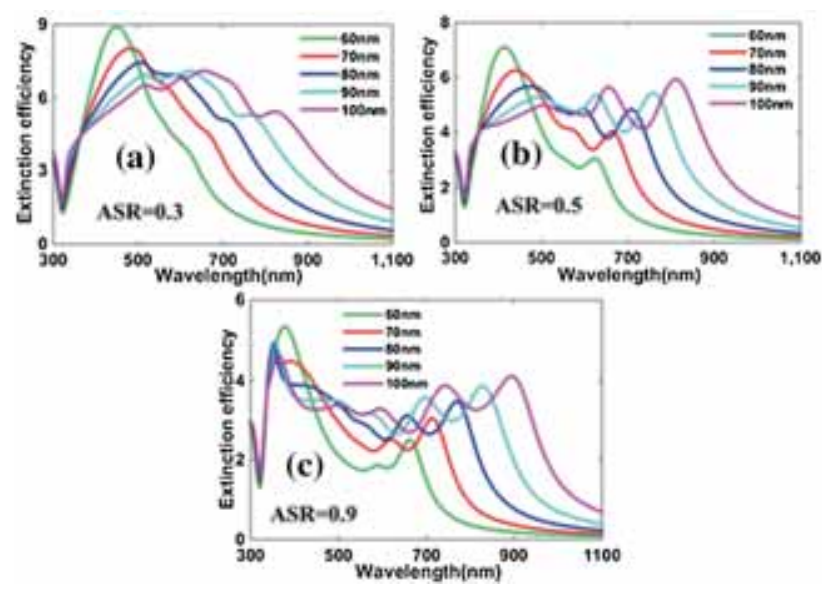

Figure 3. Extinction efficiency $Q_{\text {ext }}$ as a function of wavelength for the oblate-shaped Ag NPs for three different ASRs: (a) 0.3, (b) 0.5 and (c) 0.9, with five different effective radii of 60, 70, 80, 90 and $100 \mathrm{~nm}$ embedded in GML environment.

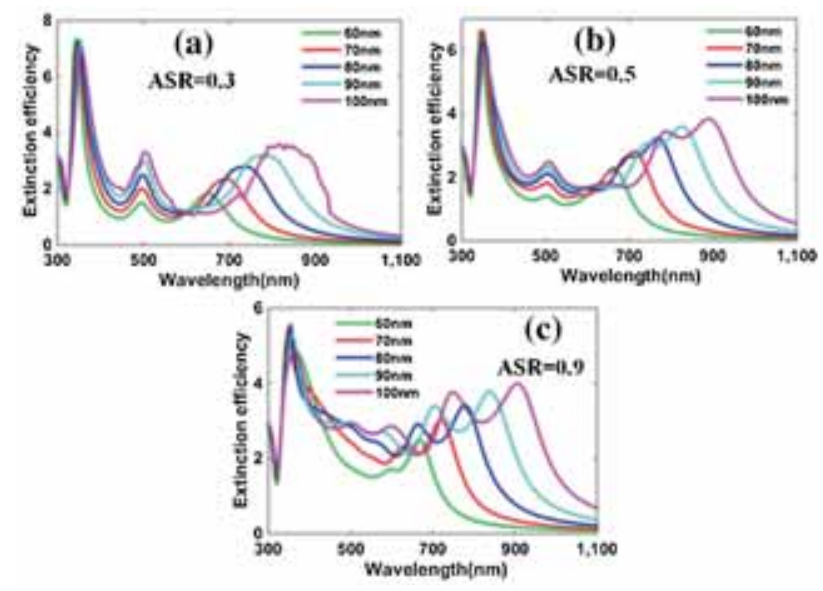

Figure 4. Extinction efficiency $Q_{\text {ext }}$ as a function of wavelength for the prolate-shaped Ag NPs for three different ASRs: (a) 0.3, (b) 0.5 and (c) 0.9, with five different effective radii of 60, 70, 80, 90 and $100 \mathrm{~nm}$ embedded in GML environment.

resonant spectra of $Q_{\text {ext }}$ at $a_{\text {eff }}=100 \mathrm{~nm}$ and ASR $=0.3$. For oblate-shaped Ag NPs the resonant peaks lying in the higher wavelength region are more tunable at ASR $=0.3$, having the magnitude of the extinction efficiency 4.146 $\left(\lambda_{\mathrm{SPR}}=600 \mathrm{~nm}\right), 4.554\left(\lambda_{\mathrm{SPR}}=665 \mathrm{~nm}\right), 5.045\left(\lambda_{\mathrm{SPR}}=\right.$ $713.4 \mathrm{~nm}), 5.353\left(\lambda_{\mathrm{SPR}}=772.5 \mathrm{~nm}\right)$ and $5.442\left(\lambda_{\mathrm{SPR}}=\right.$ $820.8 \mathrm{~nm})$ as the effective radius increases from minimum to maximum $(60-100 \mathrm{~nm})$ value. Thus, it is observed that the highly tunable resonant peaks lying in the optical broadband of $Q_{\text {ext }}$ spectrum get red-shifted as $a_{\text {eff }}$ increases up to $100 \mathrm{~nm}$ while there is a blue shift in SPR peak as the ASR increases from 0.3 to 0.9 .

Figure 4 illustrates the variation of extinction efficiency with the wavelength of the incident light for prolate-shaped
Ag NPs, by changing the ASR and effective radii of the NPs in the host medium of graphene. The prolate-shaped Ag NPs also show similar features as those of oblate-shaped Ag NPs (as shown in figure 3) with lower magnitude of optical broadband extinction efficiency $Q_{\text {ext }}$. From figure $4 a$, it can be observed that the plasmonic peak lies in higher wavelength (649-833 nm, approximately) domain having the magnitude of the extinction efficiencies as $1.847\left(\lambda_{\mathrm{SPR}}=649 \mathrm{~nm}\right)$, $2.259\left(\lambda_{\mathrm{SPR}}=686.6 \mathrm{~nm}\right), 2.787\left(\lambda_{\mathrm{SPR}}=729.5 \mathrm{~nm}\right)$, $3.192\left(\lambda_{\mathrm{SPR}}=778 \mathrm{~nm}\right)$ and $3.58\left(\lambda_{\mathrm{SPR}}=833 \mathrm{~nm}\right)$ at fixed ASR $=0.3$ with variation in $a_{\text {eff }}$ from 60 to $100 \mathrm{~nm}$. These SPR peaks are red-shifted as the $a_{\text {eff }}$ increases from 60 to $100 \mathrm{~nm}$ with tunability of $184 \mathrm{~nm}$, approximately. Further, we can see that the magnitude of these tunable resonant peaks (lies in the higher wavelength limits) gradually increases with increase in ASR as shown in figure $4 a-c$. There is a blueshift in extinction efficiency spectrum with increase in ASR because it approaches towards spherical geometry as ASR increases to its maximum value as shown in figure $4 \mathrm{a}-\mathrm{c}$.

Here, we have analysed that as the ASR increases from 0.3 to 0.9 , the individual effects of the longitudinal and the transversal resonances merge together, giving rise to the broadband spectrum of extinction efficiency. This happens because of excitation of the free electron density oscillations becoming homogenous on the surface of NPs due to the increment in ASR and ideally approaches the extinction efficiency spectra of sphere as discussed earlier (figure 1).

\section{Conclusion}

In the present study, we have used DDA to study the extinction efficiency and SPR tunability of Ag NPs of different sizes and shapes, surrounded in a graphene environment. Here, we have considered the oblate and prolate shapes of Ag NPs against the quasi-static approximation, where the effective radii of the $\mathrm{Ag}$ NPs are greater than $50 \mathrm{~nm}$ (varies from 60 to $100 \mathrm{~nm}$ ). It has been observed that high tunable longitudinal plasmonic resonances for both asymmetric (oblate and prolate)-shaped $\mathrm{Ag}$ NPs are red-shifted as the $a_{\text {eff }}$ increases from 60 to $100 \mathrm{~nm}$. In case of the oblate-shaped Ag NPs, the tunabilities of longitudinal and transverse plasmonic resonance are approximately 220 and $69 \mathrm{~nm}$, respectively; on the other hand, for the prolate shape they are approximately 184 and $12 \mathrm{~nm}$, respectively, as $a_{\text {eff }}$ increases up to its maximum limit (100 nm). The proposed metal nano-geometries (oblate and prolate) in graphene environment can be effectively used to trap incident light in the sub-wavelength regime as a requirement of the scientific community.

\section{Acknowledgement}

One of the authors (Shivani Bhardwaj) is thankful to MNRE, India, for providing the financial support for this research. 


\section{References}

[1] Atwater H A and Polman A 2010 Nat. Mater. 9205

[2] Catchpole K R and Polman A 2008 Appl. Phys. Lett. 93191113

[3] Nakayama K, Tanabe K and Atwater H A 2008 Appl. Phys. Lett. 93121904

[4] Kim S K et al. 2014 ACS Nano 83707

[5] Bermel P, Luo C, Zeng L, Kimerling L C and Joannopoulos J D 2007 Opt. Express 1516986

[6] Hideyuki I, Koichiro T, Ichiro T, Toshiaki H and Hiroki N 2000 Jpn. J. Appl. Phys. 395132

[7] Ditlbacher H, Krenn J R, Lamprecht B, Leitner A and Aussenegg F R 2000 Opt. Lett. 25563

[8] Juan M L, Righini M and Quidant R 2011 Nat. Photon. 5349

[9] Liang Z, Sun J, Jiang Y, Jiang L and Chen X 2014 Plasmonics 9859

[10] Nie S and Emory S R 1997 Science 2751102

[11] Noguez C 2007 J. Phys. Chem. C 1113806

[12] Amendola V, Bakr O M and Stellacci F 2010 Plasmonics 5 85

[13] Kelly K L, Coronado E, Zhao L L and Schatz G C 2003 J. Phys. Chem. B 107668

[14] Koppens F H L, Chang D E and García de Abajo F J 2011 Nano Lett. 113370

[15] Alsawafta M, Wahbeh M and Truong V V 2012 J. Nanomater. 201210
[16] Grand J et al. 2006 Plasmonics 1135

[17] Bhardwaj S, Pathak N K, Ji A, Uma R and Sharma R P 2017 Plasmonics 12193

[18] Bhardwaj S, Uma R and Sharma R P 2016 Plasmonics 12961

[19] Grigorenko A N, Polini M and Novoselov K S 2012 Nat. Photon. 6749

[20] Jablan M, Soljačić M and Buljan H 2013 Proc. IEEE 1011689

[21] Lu H, Cumming B P and Gu M 2015 Opt. Lett. 403647

[22] Novoselov K S et al 2005 Nature 438197

[23] Palik E D 1998 Handbook of optical constants of solids (San Diego: Academic)

[24] Johnson P B and Christy R W 1972 Phys. Rev. B 64370

[25] Sosa I O, Noguez C and Barrera R G 2003 J. Phys. Chem. B 1076269

[26] Draine B T and Flatau P J 2008 J. Opt. Soc. Am. A 252693

[27] Draine B T and Flatau P J 2013 ArXiv preprint arXiv:1305.6497

[28] Draine B T and Flatau P J 1994 J. Opt. Soc. Am. A 111491

[29] Bohren C F and Huffman D R 2008 Absorption and scattering of light by small particles (Weinheim, Germany: John Wiley \& Sons)

[30] Zhao B, Zhao J M and Zhang Z M 2014 Appl. Phys. Lett. 105 031905

[31] Wang B, Zhang X, Yuan X and Teng J 2012 Appl. Phys. Lett. 100131111

[32] García M A 2011 J. Phys. D: Appl. Phys. 44283001 